\title{
A Serious Case of Nail-Eating Pica Occurred in a Labrador Dog
}

\author{
Miss Zeyu Shi ${ }^{1}$, Hongsen $\mathrm{Su}^{2}$ and Nairui Huo ${ }^{1 *}$ \\ ${ }^{1}$ College of Animal Science and Veterinary Medicine, China \\ ${ }^{2}$ Com Pet Clinic, China
}

*Corresponding author: Nairui Huo, College of Animal Science and Veterinary Medicine, Taigu (030800), Jinzhong, Shanxi, PR China

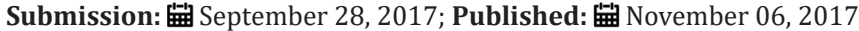

\begin{abstract}
Pica is a kind of eating disorder with nonfood substances. This case report describes a serious case of pica in which a Labrador retriever at the age of 8 weeks ate 54 nails. The corresponding clinical diagnosis is reported and the reasons of this disorder are analyzed. All nails were removed via an abdominal operation. The dog suffering pica in this case was on a balanced diet, showing that pica was not caused by malnutrition. We suggest that pet owners should give more time and accompany to their pets.
\end{abstract}

Keywords: Spica; Labrador dog; Pica; Nail-eating; Malnutrition

\section{Introduction}

Pica is characterized by special appetite for substances that are largely non-nutritive, such as paper, clay, metal, chalk, soil, glass, or sand [1]. The term itself originates from the Latin word for magpie. This pattern of eating should last for at least one month to fit the diagnosis of pica. Pica has been most widely documented in humans, and also has been reported in cats, dogs, mice and monkeys in response to poisoning [2-5]. There has been some confusion regarding whether or not pica should be classified as an eating disorder on its own, or as a symptom of other types of mental illness [6]. Complications caused by pica may occur in gastrointestinal tract, including toxicosis, constipation, and abdominal distension. For instance, wool, hair and fur may cause intestinal obstruction, dirt or dust may cause soil-transmitted parasitic infections. Sharp metal objects like nails in this report may cause injury to the esophagus, and may even cause perforation if timely treatment is not given.

There are no specific tests that can confirm pica. Veterinarian softens testiron zinc levels in blood, which might lowered because pica could occur in patients suffering malnutrition. Microbiological culture is always carried out if suspicious vomit or stool samples are collected. Blood lead level should also be checked for patients who have orally exposed to paint or other lead-based substances. Abdomen X-ray inspection is usually used for confirmation if there are high-density foreign objects in the stomach or intestines. For human individuals with autism, schizophrenia, or certain physical disorders (such as Kleine-Levin syndrome), non-nutritive substances may be eaten.
Current research on the causes of pica suggests that in many cases, the disorder is caused by a mineral deficiency or hookworm infection [7]. The substance eaten often contains the lacked mineral for the body, such as iron, in the case of pica [8]. Unlike in humans, pica in dogs or cats may be a sign of immune-mediated hemolytic anemia, especially when it involves eating substances such as mud grout, concrete dust or sand. Dogs manifesting this form of pica should be tested for anemia through a complete blood count (CBC) test or hematocrit level detection $[9,10]$. However, since increased micronutrient levels may indicate pica caused by a natural mechanism, geophagia may not be accurately diagnosed as pica since it is not actually a diseased behavior.

Treatments for pica may differ with different causes. Complementation of the corresponding nutrients or minerals (ex. Iron deficiency) is necessary to combat the disease. Moreover, poisoning caused by paint or other toxic chemical substances may be treated with gastric lavage and catharsis. If sharp metal object is eaten, and only in this case, X-ray detection is the best choice for locating the foreign objects and design a reasonable surgical corridor.

\section{Case Report}

This report deals with a rare case of nail-eating pica. A young male, Labrador dog (nicknamed“Stretch"), born May $10^{\text {th }} 2015$ (8 weeks old), was referred to the Peace Pet Hospital with a one-week history of anorexia and depression. The dog followed a regular vaccination and endoparasite and ectoparasite prevention protocol. 
The dog was found huddled up under the bed, with vomit next to him two days earlier (day 0 ). The next day vomiting disappeared temporarily and got worse on the evening, one nail and fresh blood was found in the vomit. The dog was referred to the hospital next morning.

Routine examination showed that body temperature was 38.9 ${ }^{\circ} \mathrm{C}$, slightly higher than normal $\left(37.5-38.5^{\circ} \mathrm{C}\right)$, but still at a normal level for young puppies $\left(38.5-39^{\circ} \mathrm{C}\right)$. Heartbeat was 120 beats $/ \mathrm{min}$, which is a little bit higher for young dogs, because the normal level for adults are 70-120 beats/min. Breath frequency was 37 breaths/ min, obviously higher than normal (10-30 breaths/min). In general, the above three indicators were slightly or severely increased for the young dog. Light reflection on pupils was normal. The dog carried a notable swollen lower abdomen, which upon palpation felt hard, warm and painless. Diagnostics included a biochemical test, a blood biochemical examination, a blood routine, an X-ray examination and a B-scan ultrasonography. The biochemical results showed a slight increase in GLU $(119 \mathrm{mg} / \mathrm{dl}$, normal level: $60-115 \mathrm{mg} / \mathrm{dl}$ ), which was likely due to chronic inflammation. The level of $\mathrm{HCO}_{3}$ was $26.4 \mathrm{mmol} / \mathrm{L}$, which was higher than normal $(15-23 \mathrm{mmol} / \mathrm{L})$. The BEecf level was $1 \mathrm{mmol} / \mathrm{L}$, indicating that the dog had slight alkalosis.

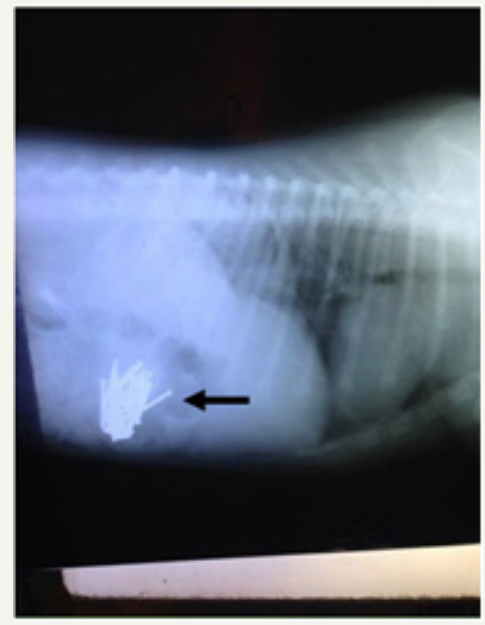

Figure 1: Sagittal reconstructed X-ray image of nails in the left stomach of the dog (black arrows).

An X-ray revealed that most of the nails were in the stomach, with the remaining nails already in the intestines (Figure $1 \& 2$ ). The dog was initially treated conservatively with a pain reliever tilidine. According to the results of X-ray, the surgery was immediately operated after an ultrasonography examination, which confirmed the definite nail position.

The dog was immobilized with a dorsal position after given a muscle relaxant atropine sulfate (Atropine sulfate is not muscle relaxant but Para sympatholytic drug to reduce vomation) $(0.1 \mathrm{mg} /$ $\mathrm{kg}$ ), then a general anesthesia was given with Shutai, a mixture of tiletamine and zolazepam, at a dosage of $15 \mathrm{mg} / \mathrm{kg}$ body weight. Abdominal hair was shaved and cleaned with disinfectant. A sterile surgical membrane was attached to the hairless abdomen.
A laparotomy sponge was spread across the abdomen and routine surgical site disinfection was performed. Along the ventral midline, a $10 \mathrm{~cm}$ incision was made from $1 \mathrm{~cm}$ behind the xiphoid to the front pubis. The abdominal wall was incised layer by layer till the stomach and the intestine were completely exposed. An incision was made to the intestine and 9 nails were taken out from the jejunum. Then the incision was cleaned with warm saline and sutured. The intestine was placed back into the abdominal cavity after being flushed with penicillin saline. Then the stomach was pulled out of the abdominal cavity and an incision was made between the greater and lesser curvature where there were fewer blood vessels. The liquid in the stomach was sucked out, and 45 nails were removed from the stomach in total. The incision was washed and stitched and the stomach was put back. The abdominal cavity was thoroughly rinsed with sterile saline, the abdominal wall and skin were sutured, the incision was disinfected and the edge was covered with a sterilized bandage.

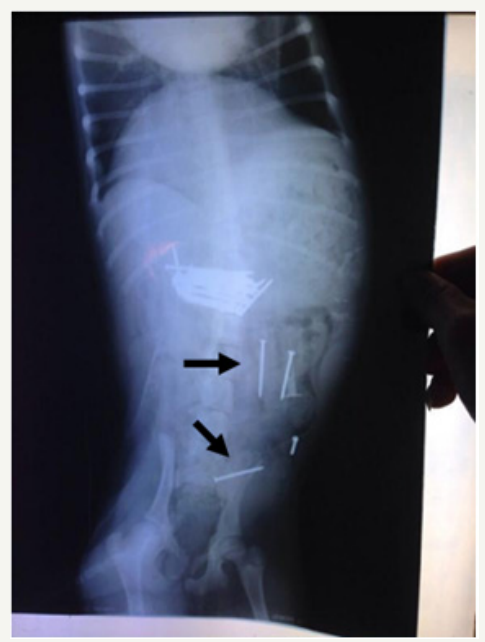

Figure 2: Sagittal reconstructed X-ray image of nails in the stomach and intestinal tract of the dog (black arrows).

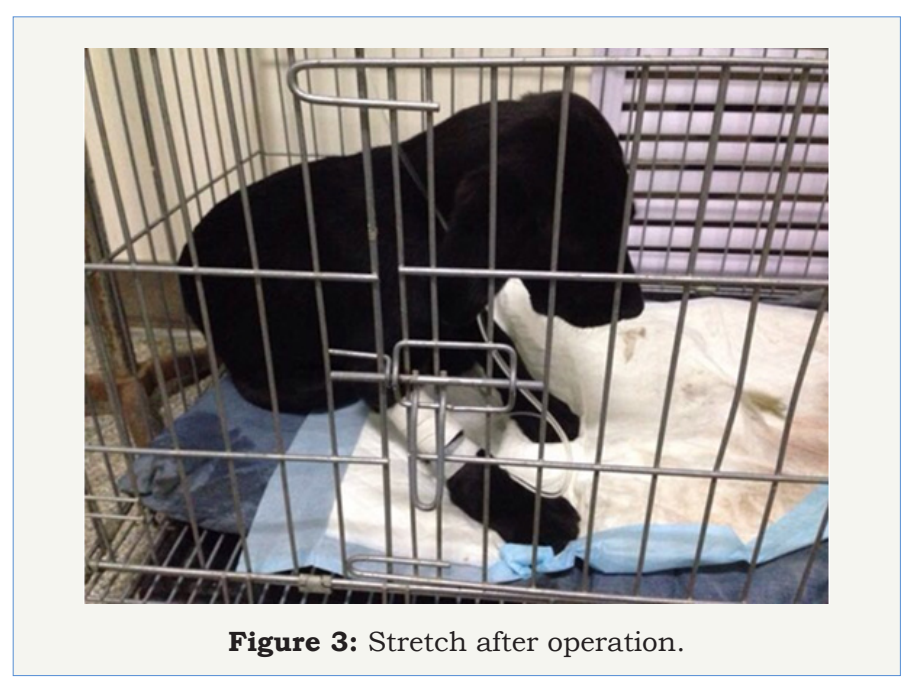

After surgery, the dog wore an Elizabethan collar for 3 days to avoid biting and licking of the surgical site, and kept in a fasting state (Figure 3). During this time, the dog was intravenously injected 
with glucose, together with rehydration therapy. By analyzing the common pathogenic bacteria in the operative site, a corresponding guideline for the preventive use of antibiotics was established. After 3 days, Stretch (the dog) was given a small amount of liquid food, and gradually transitioned to a normal diet. Postoperative infection was not found and the suture was dismantled a week after operation.

\section{Discussion}

Pica is a behavior pattern likely driven by multiple factors: age, gender, religion, culture, genetics, psychosocial state, and nutritional needs. For pets, two of the most common reasons are lack of nutrition or accompany. Nutritional theories are most commonly cited, often linking pica to deficiencies in minerals such as iron and zinc [11]. Therefore, a mineral or vitamin supplement is necessary after surgery. Pica has also been significantly associated with an increased likelihood of not having a strong and supportive relationship with family, a lack of social contact, an absence of participation in activities of long-standing interest, absence of involvement in a day program, as well as a lack of involvement in recreational activities, but has not been related to interpersonal conflicts [12]. Stretch (the dog) case was likely caused by a lack of contact with owners. The owner said when they go to work, Stretch is locked in a cage for more than $8 \mathrm{~h}$ each day. It is important to be aware of this common, but commonly missed condition. This type of situation might make young dogs feel hopeless and alone. Many research works indicated that pica is related to neuropsychopharmacology or biological psychiatry probably caused by stress [13].

The clinical consequences of pica may have broad epidemiological implications such as intoxication and geophagia in pets. The worst acute and chronic medical complications posed are surgical emergencies (intestinal obstruction from bezoars). The reported subtle encroaching symptom includes parasitosis, intoxication, and nutritional deficits [14]. Intestinal obstructions often occur in those with pica. Gastrointestinal parasites can occur more frequently in individuals with dirt-eating or fecal-eating [15], but no obvious parasitic infections were noted in this case.

The prevalence of pica is not known [11]. It is difficult to establish prevalence because of differing definitions and the reluctance of patients to admit to abnormal cravings and ingestion. In pets, pica is probably due to the lack of certain nutrients such as vitamins and minerals and can be intervened with nutritional supplements. For pets with chaotic diets and lack of accompany, we suggest that owners spend more time with them and pay more attention to the pet behavior so that the pets can avoid the occurrence of pica.
Many different treatment regimens have been described, with variable responses. Conservative treatment can be used when a case is not serious, or the foreign object is soft and small, which can not cause further damage. Emergency surgery is needed when a pet swallows foreign objects that are sharp and capable of stabbing the gastrointestinal tract, or when the objects cause lifethreatening problems such as acute intestinal obstruction. The location of foreign objects can be determined through X-rays in order to choose an appropriate surgical pathway. For cases in which there is evidence of damage to the digestive tract, the damaged areas should be repaired. In the case of complications like anorexia, apastia, oremesis, different treatments can be used depending on the cause. Postoperative observation of the pet and maintenance is a must.

\section{References}

1. López LB, Ortega SCR, de Portela ML (2004) Pica during pregnancy: a frequently underestimated problem. Arch Latinoam Nutr 54(1): 17-24.

2. Santucci D, Corazzi G, Francia N, Antonelli A, Aloe L, et al. (2000) Neurobehavioral effects of hyper gravity conditions in adult mouse. NeuroReport 11(15): 3353-3356.

3. Walker AR, Walker BF, Jones J, Verardi M, Walker C (1985) Nausea and vomiting and dietary cravings and aversions during pregnancy in South African women. Br J Obstet Gynaecol 92(5): 484-489.

4. Houpt K (1982) Ingestive behavior problems of dogs and cats. Vet Clin North Am Small Anim Pract 12(4): 683-692.

5. Krishnamani R, Mahaney WC (2000) Geophagy among primates: adaptive significance and ecological consequences. Anim Behav 59(5): 899-915.

6. Parry-JB, Parry-JWL (1992) Pica: symptom or eating disorder? A historical assessment. Br J Psychiatry 160: 341-354.

7. Lumsden WHR (1979) Advances in parasitology. Academic Press 17: 337.

8. Sayetta RB (1986) Pica: an overview. Am Fam Physician 35(5): 181-185.

9. Plunkett, Signe J (2000) Emergency procedures for the small animal veterinarian. Elsevier Health Sciences p. 11.

10. Feldman BF, Zinkl JG, Jain NC (2000) Schalm's veterinary hematology. Blackwell Publishing, UK, England, p. 506.

11. Rose EA, Porcerelli JH, Neale AV (2000) Pica: common but commonly missed. J Am Board Fam Pract 13(5): 353-358.

12. Ashworth M, Hirdes JP, Martin L (2009) The social and recreational characteristics of adults with intellectual disability and pica living in institutions. Res Dev Disabil 30(3): 512-520.

13. Hergüner S, Ozyildirim I, Tanidir C (2008) Is pica an eating disorder or an obsessive-compulsive spectrum disorder? Prog Neuro-Psychoph 32(8): 2010-2011.

14. Blinder BJ, Salama C (2008) An update on pica: prevalence, contributing causes, and treatment. Psychiatric Times 25(6).

15. Danford DE, Huber AM (1982) Pica among mentally retarded adults. Am J Ment Defic 87(2): 141-146. 